\title{
Relapse of hyperthyroidism after hemithyroidectomy in concurrent medullary thyroid cancer and Graves' disease
}

\author{
Meng ZW ${ }^{1}$, Zhang $\mathrm{YJ}^{2}$, Li W' ${ }^{1}$, Shi $\mathrm{T}^{2}$, Wu $\mathrm{SG}^{3}$, Tan $\mathrm{J}^{1}$ \\ Department of Nuclear Medicine, Tianjin Medical University General Hospital, Tianjin, P.R. China. \\ james_mencius@hotmail.com
}

\begin{abstract}
We present a rare case of concurrent medullary thyroid cancer (MTC) and relapse of Graves' disease (GD). A 26-year-old Chinese female suffered from GD for 1 year. Physical examination demonstrated mildly diffuse goiter with a palpable $1.0 \mathrm{~cm}$ nodule in the right thyroid lobe and exophthalmos. Before hemithyroidectomy, calcitonin was elevated and thyroglobulin was normal. After surgery, histopathological findings proved coexistence of MTC and GD. 16 months later, GD relapsed and radioactive iodine 131 therapy was given. Meanwhile, postoperative monitoring showed well controlled calcitonin and carcinoembryonic antigen. Our lesson demonstrates although concomitant of MTC and GD is very rare, yet after hemithyroidectomy for the single nodular MTC lesion, remnant thyroid lobe could be the source of GD relapse very soon. More importantly, the relapse of GD and the following radioiodine therapy and radiation exposure could have been prevented if a better therapy plan of total thyroidectomy was adopted in the first place, instead of hemithyroidectomy (Tab. 1, Fig. 2, Ref. 11). Full Text in PDF www.elis.sk.

Key words: medullary thyroid cancer, Graves' disease, relapse, calcitonin, thyroidectomy.
\end{abstract}

The concurrency of thyroid cancer with Graves' disease (GD) is very rare, occurring in $0.8-8.1 \%$ of glands removed in the treatment of GD (1-4). Medullary thyroid cancer (MTC) is an uncommon and challenging malignancy, which accounts for only about $4 \%$ of all thyroid cancer cases (1). We conducted a systematic literature search on PubMed database for articles in any language on the concomitancy of MTC and hyperthyroidism published from 1950 to 2010 . We retrieved only 12 cases of concomitant MTC with hyperthyroidism (2-11), yet relapse of GD after surgical treatment of MTC has not been documented before. Here we report the 13th case, a 26-year-old Chinese female, who was the youngest and 1st such patient with GD relapse with in depth analysis of histopathology and immunohistochemistry.

\section{Case report}

This Chinese female patient was initially diagnosed with GD when she was 25 in another hospital. We could still retrieve her

${ }^{1}$ Department of Nuclear Medicine, Tianjin Medical University General Hospital, Tianjin, P.R. China, ${ }^{2}$ Department of Pathology, Tianjin Medical University General Hospital, Tianjin, P.R. China, and ${ }^{3}$ Department of General Surgery, Tianjin Medical University General Hospital, Tianjin, P.R. China

Address correspondence: ZW Meng, MD, PhD, Department of Nuclear Medicine, Tianjin Medical University General Hospital, Anshan Road No. 154, Heping District, Tianjin, 300052 P.R. China.

Phone: +86.13132038325 , Fax: +86.022 .27813550$

Acknowledgement: This investigation was partially supported by China National Natural Science Foundation grant 30900376, Key Project of Tianjin Science and Technology Committee Foundation grant 10JCZDJC19000, Tianjin Medical University Scientific Research grant 2008KY20 and Tianjin Medical University New Century Excellent Talents Program (awarded to Zhaowei Meng). laboratory results at that time. She had elevated levels of triiodothyronine (T3, 648.0 ng/dL, reference 84.6-201.8), thyroxine (T4, $21.93 \mu \mathrm{g} / \mathrm{dL}$, reference 5.13-14.06), free T3 (FT3, $27.90 \mathrm{pg} /$ $\mathrm{dL}$, reference 1.82-4.62) and free T4 (FT4, $7.91 \mathrm{ng} / \mathrm{dL}$, reference $0.93-1.71)$, yet suppressed level of thyrotropin $(0.01 \mu \mathrm{IU} / \mathrm{mL}$, reference $0.27-4.20$ ). She was given regular methimazole treatment for 1 year before she came to our hospital. Her past medical history was not significant. Physical examination demonstrated mildly diffuse goiter with a palpable $1.0 \mathrm{~cm}$ nodule in the right thyroid lobe and exophthalmus. Laboratory testing showed nor-
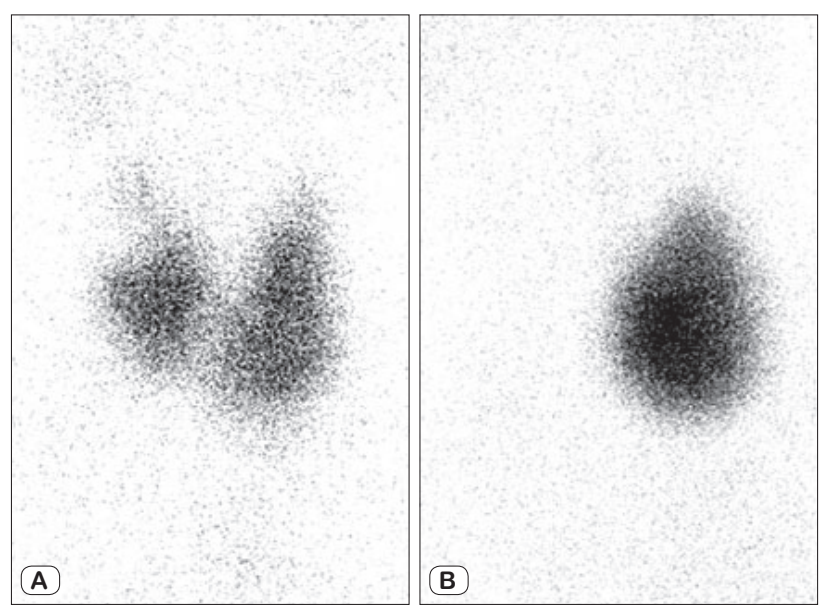

Fig. 1. A) $30 \mathrm{~min}$ after intravenous injection of technetium-99m pertechnetate, pre-surgical thyroid scan was performed, which demonstrated a cold nodule in the upper part of right thyroid lobe. B) 18 months after the operation when hyperthyroidism relapsed, a second thyroid scan was performed, which showed an enlarged left thyroid lobe with homogeneously increased uptake ability. 
Tab. 1. Features of the published cases of concurrent MTC and hyperthyroidism.

\begin{tabular}{|c|c|c|c|c|}
\hline Author & Age (year)/Sex & Tumor size $(\mathrm{cm})$ or TNM & Presurgical Ct (pg/mL) & Cause of hyperthyroidism \\
\hline Pradeep (2) & NA & NA & NA & Graves' disease \\
\hline Chao (3) & NA & NA & NA & Graves' disease \\
\hline Rieger (4) & $61 / \mathrm{M}$ & T1 N0 M0 & NA & Toxic multinodular goiter \\
\hline Rieger (4) & $67 / \mathrm{F}$ & T1 N0 M0 & NA & Toxic multinodular goiter \\
\hline Habra (5) & $70 / \mathrm{M}$ & $1.1 \mathrm{~cm}$ & $18300 \mathrm{pg} / \mathrm{mL}$ & Graves' disease \\
\hline Brandle (6) & $50 / \mathrm{M}$ & $1.0 \mathrm{~cm}$ & $4572 \mathrm{pg} / \mathrm{mL}$ & Graves' disease \\
\hline Brandle (6) & $33 / \mathrm{F}$ & $1.4 \mathrm{~cm}$ & $1300 \mathrm{pg} / \mathrm{mL}$ & Graves' disease \\
\hline McFarland (8) & $30 / \mathrm{F}$ & $1.2 \mathrm{~cm}$ & NA & Graves' disease \\
\hline Mazziotti (9) & $30 / \mathrm{F}$ & $1.6 \mathrm{~cm}$ & $5125 \mathrm{pg} / \mathrm{mL}$ & Graves' disease \\
\hline Schwartz (10) & $40 / \mathrm{F}$ & $0.5 \mathrm{~cm}$ & NA & Graves' disease \\
\hline Small (11) & 44/M & $\mathrm{T} 4$ & NA & Toxic multinodular goiter \\
\hline Current report & $26 / \mathrm{F}$ & $1.4 \mathrm{~cm}$ & $454 \mathrm{pg} / \mathrm{mL}$ & Graves' disease \\
\hline
\end{tabular}

TNM: Tumor, lymph node and metastasis

NA: Not available
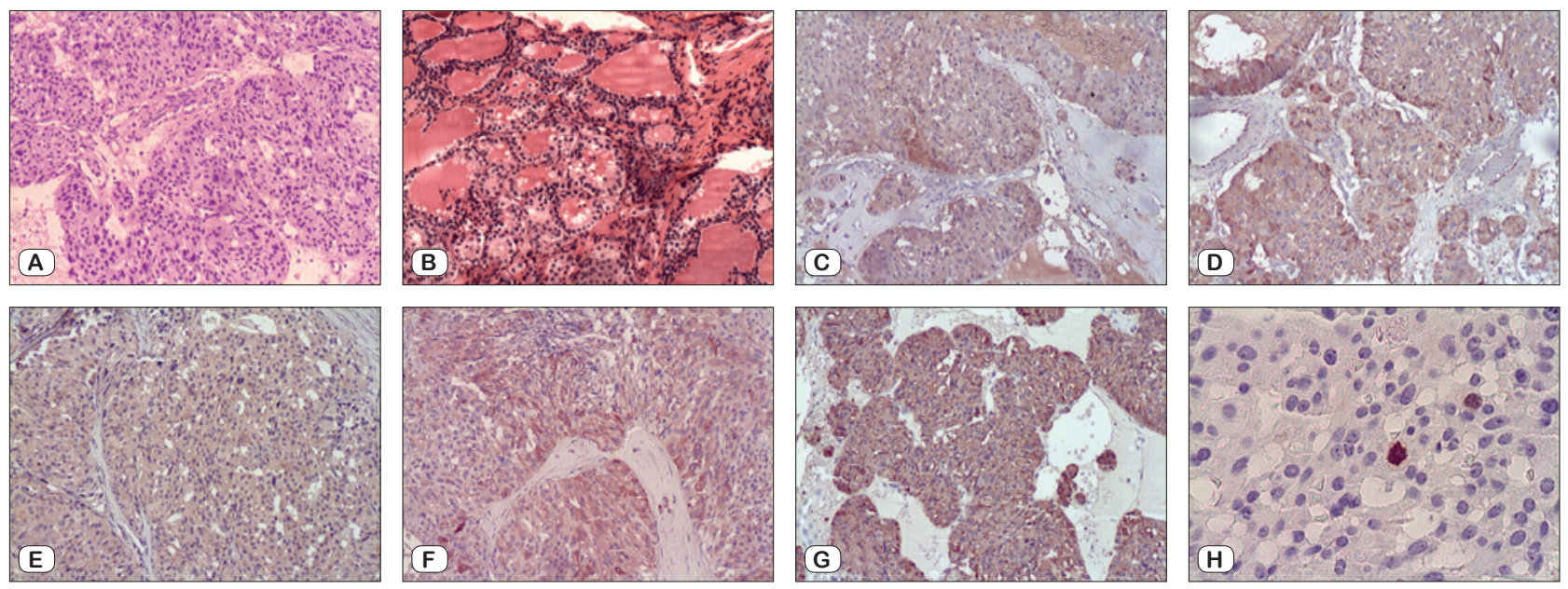

Fig. 2. A) Pathologically, hematoxylin and eosin stained section of the nodule demonstrated that the tumor cells with small, round hyperchromatic nuclei were moderately differentiated and were forming in shapes of nests or sheets. Morphologic features were suggestive to the diagnosis of MTC (magnification $\times 40$ ). B) Hematoxylin and eosin stained other areas of the right thyroid lobe showed follicles lined by tall columnar epithelial cells and "scalloped" appearance on the edges of the colloid lumens, which were typical microscopic features of GD in histopathology (magnification $\times 40$ ). C) Immunohistochemistry staining of the cancer lesion was positive for $\mathrm{Ct}$ (magnification $\times 40$ ). D) Immunohistochemistry staining of the cancer lesion was positive for chromogranin (magnification $\times \mathbf{4 0}$ ). E) Immunohistochemistry staining of the cancer lesion was positive for synaptophysin (magnification $\times 40$ ). F) Immunohistochemistry staining of the cancer lesion was positive for cytokeratin (magnification $\times 40$ ). G) Immunohistochemistry staining of the cancer lesion was strongly positive for CEA (magnification $\times 40$ ). H) Immunohistochemistry staining of the cancer lesion was sporadic positive for Ki-67 (magnification $\times 200)$.

mally controlled FT3 (5.2 pmol/L, reference 3.5--6.5), FT4 (14.9 $\mathrm{pmol} / \mathrm{L}$, reference $11.5-23.5)$ and thyrotropin $(3.69 \mu \mathrm{IU} / \mathrm{mL}$, reference $0.20-5.00)$. Her preoperative calcitonin $(\mathrm{Ct})$ was $453.8 \mathrm{pg} /$ $\mathrm{mL}$ (reference $<100.0$ ), yet her thyroglobulin $(\mathrm{Tg})$ was $9.14 \mathrm{ng} /$ $\mathrm{mL}$ (reference $<25.00$ ). Ultrasound showed a $1.5 \times 1.0 \times 1.0 \mathrm{~cm}^{3}$ inhomogeneous nodule in the upper part of right thyroid lobe with left lobe of $1.8 \times 2.1 \times 5.1 \mathrm{~cm}^{3}$ and right lobe of $2.2 \times 2.3 \times 5.8 \mathrm{~cm}^{3}$, yet no enlarged lymph nodes were detected. Thyroid scan with technetium-99m pertechnetate (Fig. 1A) showed a cold nodule in the upper part of right thyroid lobe.

Right lobe hemithyroidectomy was performed, and the nodule was found to be a $1.4 \times 1.0 \times 0.8 \mathrm{~cm}^{3}$ gray-red lesion. Lymph node dissection of the right and central neck was also performed. Pathologically, the lesion demonstrated MTC cells forming in shapes of nests or sheets (Fig. 2A) with intact capsule. In addition, non-neoplastic parenchyma of the resected thyroid lobe exhibited typical GD features (Fig. 2B). By immunohistochemistry (Figs $2 \mathrm{C}-\mathrm{H})$, the tumor manifested positivity for $\mathrm{Ct}$, chromogranin, synaptophysin and cytokeratin, and strong positivity for carcinoembryonic antigen (CEA), yet sporadic positivity for Ki-67. However, immunoreactivities for $\mathrm{Tg}$ and thyroid transcription factor-1 were negative. No lymph node metastases were detected. Because malignancy was revealed by pathology, a second surgery of complete thyroidectomy was discussed with the patient. Yet, the patient declined.

After hemithyroidectomy, she was given a regimen of 62.5 $\mu \mathrm{g}$ levo-thyroxine to obtain euthyroidism. For 15 months, her thyroid function was within normal ranges with Ct between 19.6 to $24.9 \mathrm{pg} / \mathrm{mL}$ and CEA between 0.2 to $1.2 \mathrm{pg} / \mathrm{mL}$ (reference $0.0-5.0$ ). Ultrasound monitoring of the thyroid showed nega- 
tive findings. Nevertheless, 16 months later, nervousness, tremor, palpitation, weight loss despite increased appetite and increased sweating re-appeared. At that time, FT3, FT4 and thyrotropin were $22.0 \mathrm{pmol} / \mathrm{L}, 49.6 \mathrm{pmol} / \mathrm{L}$ and $<0.01 \mu \mathrm{IU} / \mathrm{mL}$, respectively. Levo-thyroxine was withdrawn immediately. During the following 2 months, her thyroid function decreased to some extent, yet still maintained at a high FT3 level of $10.4 \mathrm{pmol} / \mathrm{L}$ and FT4 level of $38.0 \mathrm{pmol} / \mathrm{L}$, and a suppressed thyrotropin level of $0.01 \mu \mathrm{IU} /$ $\mathrm{mL}$. Ultrasound demonstrated an enlarged left thyroid lobe of $2.2 \times 3.0 \times 5.9 \mathrm{~cm}^{3}$ in size. 24 -hour thyroid uptake rate of iodine was $56.5 \%$ (reference 10-40), and thyroid scan with technetium-99m pertechnetate (Fig. 1B) showed an enlarged left thyroid lobe with homogeneously increased uptake ability. At this time Ct, CEA and Tg were $17.9 \mathrm{pg} / \mathrm{mL}, 0.7 \mathrm{pg} / \mathrm{mL}$ and $2.3 \mathrm{ng} / \mathrm{mL}$, respectively. She had a normal serum calcium, parathormone, and 24-hour urinary excretion of metanephrines, catecholamines and vanillylmandelic acid. GD relapse was diagnosed, and $6 \mathrm{mCi}$ of radioactive iodine $131\left({ }^{131} \mathrm{I}\right)$ was prescribed. After this therapy, euthyroidism was achieved within 3 months, and close follow-up was uneventful.

\section{Discussion}

MTC is a rare tumor derived from the parafollicular C cells of the thyroid. The simultaneous occurrence of thyroid cancer and hyperthyroidism varied from the published studies, while MTC was much less frequently found. We performed a systematic literature search on MEDLINE from 1950 to 2010 without language limitation. Key words were set as the combination of medullary thyroid cancer and Graves' disease or toxic goiter or hyperthyroidism. Only 12 cases with such rare entities were retrieved (Tab. 1) (2-11). Our patient was the youngest, and also the 1 st one who experienced GD relapse. Pre-surgically, we adopted serum $\mathrm{Ct}$ and $\mathrm{Tg}$ measurements as routine diagnostic evaluations of thyroid nodules. In the surgical management of the present case, hemithyroidectomy was performed. According to the MTC guideline (1), when a patient presents with a well circumscribed singular thyroid nodular lesion without enlarged lymph nodes, hemithyroidectomy could be an option, which could decrease the possibilities of complications. After surgery, histopathology was explored extensively, which confirmed the diagnosis of concomitant MTC with GD.

The merits of this case report probably exist in the discussion about the major limitations in the managements and lessons that should be learned from the case. First, during preoperative diagnostics fine-needle aspiration and RET mutation analysis were not performed. This is partly due to the inexperienced pathologists of our institution in these particular fields. Second, it would be very reasonable to argue that for this case, total thyroidectomy should be performed under the constellation in the MTC guideline, and a total thyroidectomy could clarify the nodule and cure GD in one step. However, adding the fact that there was a single thyroid nodule without enlarged lymph node, and the patient also suffered from GD with exophthalmus, we adopted a rather incomprehensible approach of hemithyroidectomy with a lower rate of complications initially. Since no lymph node metastases were found and postoperative $\mathrm{Ct}$ was well controlled, plus a second surgery was declined based on the patient's willingness, a further complete thyroidectomy was foregone. Nevertheless, relapse of GD happened so quickly after merely 16 months, although no signs of MTC relapse or metastasis appeared. Therefore, the main lesson learned from this case is that the relapse of GD and the following radioiodine therapy and radiation exposure could have been prevented if a better therapy plan of total thyroidectomy was adopted in the first place, instead of hemithyroidectomy.

\section{References}

1. Kloos RT, Eng C, Evans DB, Francis GL, Gagel RF, Gharib H, Moley JF, Pacini F, Ringel MD, Schlumberger M, Wells SA, Jr. Medullary thyroid cancer: management guidelines of the American Thyroid Association. Thyroid 2009; (19): 565-612.

2. Pradeep PV, Agarwal A, Baxi M, Agarwal G, Gupta SK, Mishra SK. Safety and efficacy of surgical management of hyperthyroidism: 15year experience from a tertiary care center in a developing country. World J Surg 2007; (31): 306-312; discussion 313.

3. Chao TC, Lin JD, Chen MF. Surgical treatment of thyroid cancers with concurrent Graves disease. Ann Surg Oncol 2004; (11): 407-412.

4. Rieger R, Pimpl W, Money S, Rettenbacher L, Galvan G. Hyperthyroidism and concurrent thyroid malignancies. Surgery 1989; (106): 6-10.

5. Habra MA, Hijazi R, Verstovsek G, Marcelli M. Medullary thyroid carcinoma associated with hyperthyroidism: a case report and review of the literature. Thyroid 2004; (14): 391-396.

6. Brandle M, Galeazzi RL, Diener PA, Schmid C. Medullary thyroid carcinoma in Graves' disease. Clin Endocrinol (Oxf) 1999; (50): 545-546.

7. Nakamura S, Saio Y, Ishimori M, Shima H. Incidental medullary thyroid carcinoma in a case of Graves' disease. Intern Med 2002; (41): 323-324.

8. McFarland KF, Hawksley VC, Reynolds JC. Hyperthyroidism and medullary carcinoma of the thyroid. South Med J 1980; (73): 1661-1663.

9. Mazziotti G, Rotondi M, Manganella G, Franco R, Capone P, Colantuoni V, Amato G, Carella C. Medullary thyroid cancer, papillary thyroid microcarcinoma and Graves' disease: an unusual clinical coexistence. J Endocrinol Invest 2001; (24): 892-896.

10. Schwartz RW, Kenady DE, Bensema M, McGrath PC, Flueck J. Medullary thyroid cancer and Graves' disease. Surgery 1989; (105): 804-807.

11. Small PK, Smith D. Sporadic medullary thyroid carcinoma associated with toxic multinodular goitre. J R Coll Surg Edinb 1997; (42): 199-200.

Received July 31, 2011. Accepted November 30, 2011. 